\title{
A Review on Dental Radiographic Images
}

\author{
*A.J.Solanki ${ }^{1}$, P.M.Mahant ${ }^{2}$ \\ ${ }^{12}$ Lecturer, B\&B Inst. Of tech., Vallabh vidyanagar \\ Corresponding author: A.J.Solanki
}

\begin{abstract}
Medical image processing is essential in many fields of medical research and clinical practices because it greatly facilitates early detection and diagnosis of diseases. This paper surveys an add-on approach in the area of medical image analysis for diagnosis of diseases in oral radiology using dental Xrays in dentistry. In case of medical images human involvement and perception is of prime importance. It is indeed a difficult task to interpret fine features in various contrast situations. The raw data obtained directly from X-ray acquisition device may yield a comparatively poor image quality representation. Because of the role of a human (dentist) interpretation based on his knowledge, experience and perception which may differ from doctor to doctor; there are chances of error in deciding the right medical treatment. Software developers along with domain experts have designed various standardized and scientific tools to minimize the human error in the case of deciding the right treatment on the basis of visual perception. One of the aims of this paper is to focus on the extracted part of the tooth from digital dental X-ray, finding the required information in the form of features and helping the dentist in the form of pre-diagnosis suggestions at an early stage.
\end{abstract}

Keywords: Dental radiograph ( $X$-ray ), dentistry, image enhancement, segmentation, feature extraction, Dental caries, Teeth segmentation, Horizontal and Vertical projection profile, Gap Valley.

\section{INTRODUCTION}

Potential benefits of imaging studies include diagnosis of illness, and the severity or benign nature of that process, is made quickly and accurately. Medical imaging is essential not only at initial diagnosis, but for monitoring how the disease is responding to treatment or if the disease is progressing, and when a treatment plan might be stopped or adjusted. Our technologies are fundamental to standards of care. By catching disease early, reducing the need for invasive, inpatient procedures and facilitating shorter recovery times, medical imaging saves money and improves efficiency in the health care system.It is increasingly playing a vital role in areas like ophthalmology, cardiology, gynaecology, orthopaedics, dentistry and neurology. In the recent years, different techniques of processing on image have been actively used for the diagnosis of oral diseases in dentistry. There are various diagnostic methods for dentistry which include, Computed Axial Tomography (CT Scan or CAT scan), Ultrasonography (US), Panoramic Imaging, Intra Oral and Extra Oral Radiography and MRI. These imaging systems are helpful in confirming the different types of dental disease infections. By using the radiographs of teeth, experts can found the number of diseases such as Fracture of tooth, Abrasion, Dental Caries, Attrition, Gingivitis,
Periodontal, Abscess, Interdental bone Loss, Supernumerary Teeth, Impacted teeth, Cysts, Malignancies, Developmental defects, Future Malocclusion etc.

\subsection{Digital Dental Radiograph (X-rays)}

Dental Radiographs (X-rays) are a type of picture of the teeth and mouth. X-rays are a form of electromagnetic radiation, just like visible light. They are of higher energy and can penetrate the body to form an image on film. Structures that are dense (such as silver fillings or metal restoration ) block most of the photons and appear white on developed film. Structures containing air appear as black on film while teeth, tissue, and fluid appear as shades of gray. An x-ray is a type of energy that passes through soft tissues and is absorbed by dense tissue. Teeth and bone are very dense, so they absorb $\mathrm{X}$-rays, while $\mathrm{X}$-rays pass more easily through gums and cheeks.Dental X-rays help to find problems with the teeth, mouth, and jaw. Dental X-ray pictures can show cavities, hidden dental structures (such as wisdom teeth), and bone loss that cannot be seen during a visual examination. They are very useful in detecting the early stages of decay between teeth. Compared to traditional X-rays, only half the dosage of radiation is needed for obtaining a dental X-rays of comparable quality. They do not require time for film development, so dentists need to wait for only a 
few seconds before the acquired image is displayed. Dentists can take another image instantly if the acquired image is not of good quality, so in general digital dental X-rays in a patient's record have better image quality than conventional dental X-rays. Mainly due to their advantages in speed, storage, and image quality, digital dental X-rays are now routinely used. Here are some benefits of digital radiography such as the image quality is very high which helps to identify the diseases, it is faster and easier to use as compared with conventional method, also it requires lower dosage of radiation and no chemicals for developing.

\subsection{Types of Dental Radiographs}

Intraoral X-rays are the most common type of radiograph taken in dentistry. They give a high level of detail of the tooth, bone and supporting tissues of the mouth. These X-rays allow dentists to:

- Find cavities

- Look at the tooth roots

- Check the health of the bony area around the tooth

- Help diagnose periodontal disease

- See the status of developing teeth

\subsubsection{The Bitewing X-ray}

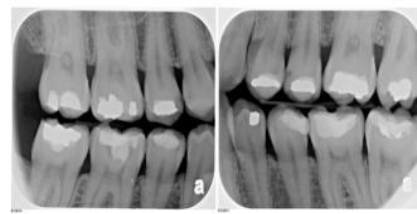

(a)

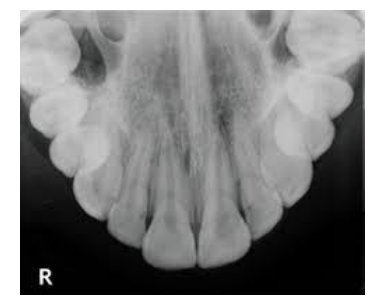

(c)
It highlights the crowns of the back teeth. Dentists take one or two bite-wing X-rays on each side of the mouth. Each X-ray shows the upper and lower molars (back teeth) and bicuspids (teeth in front of the molars). These X-rays are called "bite-wings" because you bite down on a wing-shaped device that holds the film in place while the X-ray is taken. These X-rays help dentists find decay between back teeth. Figure 1(a) shows bitewing view.

\subsubsection{Periapical X-ray}

It highlights only one or two teeth at a time. It shows the entire length of each tooth, from crown to root as shown in figure 1(b). This used to determine teeth caries in a particular tooth, because it allows a dentist to visualize the whole tooth as well as the teeth surrounding cavities of bone.

\subsubsection{Occlusal View}

They are larger than most X-rays. They highlight tooth development and placement in children. Each $\mathrm{X}$-ray shows nearly the full arch of teeth in either the upper or lower jaw. The film rests on the biting surface of the teeth.

\subsubsection{Panoramic View}

Panoramic X-rays shows the whole teeth structure with jaws and teeth in single view. These type of $\mathrm{X}$ rays are used to detect different infections or problems present in teeth such as cysts, fractures, tumors, impacted teeth and dental caries etc.

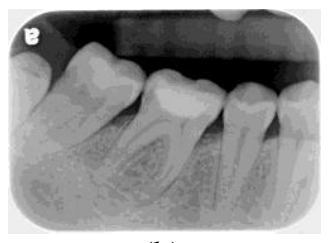

(b)

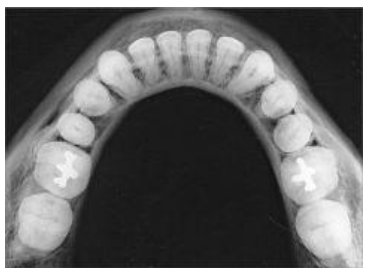

(c)

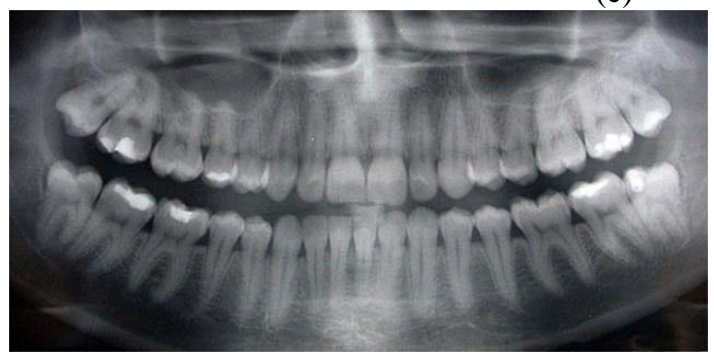

(d)

Fig 1 a): Bitewing View b): Periapical View c):Occlusal View d): Panoramic View

\subsection{Basics of Tooth Structure}

\section{BACKGROUND}

The studies of human tooth structures come under the field of dental anatomy. The teeth are made up of a group of hard substances present in the oral cavity. Teeth are generally used to masticate the food, provide shape to mouth and also used in producing the speech. the main parts of the tooth are crown and root. Each tooth is an organ consisting of three layers: Enamel, Dentin and Pulp
- Enamel: Enamel is the hardest and most highly mineralized substance of the body. It is outermost white colour part of teeth and made up of calcium phosphate. It can be damaged by decay if teeth are not cared properly.

- Dentin: It is a teeth layer lies just below the enamel. It is made of living cells, which secrete a hard mineral substance. Because it is softer than enamel, it decays more rapidly and is subject to severe cavities if not properly treated, but dentin still 
acts as a protective layer and supports the crown of the tooth.

- Pulp: The softer, living inner structure of teeth. Blood vessels and nerves run through the pulp of the teeth. If tooth decay reaches the pulp, you usually feel pain and may require a root canal procedure to be done.

Basic tooth structure is shown in figure 2 .

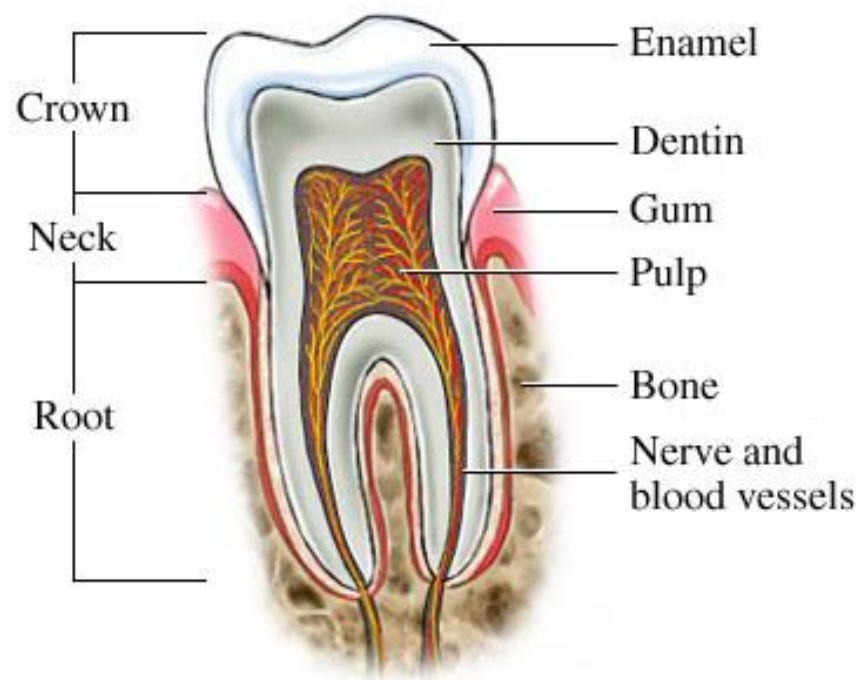

Fig 2: Basic Tooth Structure

\subsection{Types of Diseases \\ 2.2.1 Enamel Caries:}

In dental X-ray images, enamel caries can be recognized by a loss on the interproximal surfaces of the enamel. To be detectable on a radiographic image there must be a $30 \%$ to $50 \%$ change in the mineral content of the enamel lesion.

\subsubsection{Dentinal Caries}

Dentinal Caries can be recognized by noting the focal loss of dentinal radiopacity. Dentin caries may be discerned inter- proximally, on the occlusal surface, buccally/lingually, or on root surfaces.

\subsubsection{Pulpitis}

Dental Caries, commonly known as tooth decay or cavities extends below the tooth dentin and it affects the nerves or the blood vessels, the it is known as the pulpitis.

\section{REVIEW}

is an art and science of diagnosis and treatment of diseases and disorders of the oral cavity and its associated structures. The components of dentistry includes, periodontics, oral pathology, orthodontics, oral and maxillofacial surgery, pedodontics, prosthodontics, forensic odontology, geriatric dentistry and dental implantology. Periodontics deals with diseased gums, Oral pathology concentrates in the diagnosis, Orthodontics aids in the correction of mal-aligned teeth, oral and maxillofacial surgery is concerned with major surgical procedures related to the dental and associated structures, pedodontics deals with children and prosthodontics accounts for the rehabilitation process . Forensic odontology consists of the gathering and the use of dental evidence in human identification that is primarily documenting and verification of identity . Geriatric dentistry is the delivery of dental care to senior adults involving the diagnosis and treatment of problems associated with age related diseases. Dental implantology is a recent milestone in dentistry and in this method the dental images play a vital role in the selection and correct placement of implants. Common dental diseases are tooth decay and gum diseases. Other dental related disorders include, discolored front tooth, fractured teeth from trauma, over-retained primary teeth, abnormal eruption of wisdom teeth, tempero-mandibular joint disorders and pathological lesions like dental cyst, plague, cancer and tumor. In recent years there is increasing awareness among people about the fact that the dental problems can cause other serious health implication. Prevention and early treatment of dental diseases may contribute to overall health. The conventional diagnostic procedures include tracing the history, measurement of pocket depth and clinical attachment level, and assessment of tooth mobility and displacement, and the increase in local temperature. The goal of this research is to automate the process of representation and extracting different features of dental x-ray images to use in further applications.

\subsection{Existing Systems}

Dr. C. K. Modi proposed feature extraction technique for dental X-ray image. There are different features for radiographic image such as 
shape and texture. For shape analysis Fourier descriptor features and to describe texture analysis of the extracted tooth GLCM properties such as contrast, Energy, Correlation and Homogeneity are used. Also the author suggested future work in the given system is finding a novel feature extraction technique which explores the geometry of teeth which remains inherently unique to each individual [3]. Shape extraction algorithm is developed using contour information and mahalanobis distance measure is done for matching dental records. Integral intensity projections is used to segment upper and lower jaw and also to segment individual tooth. Shape extraction is improved by fast connected component labeling [4]. Grayscale contrast stretching transformation to improve the performance of teeth segmentation, also offered a mathematical morphology approach to the problem of teeth segmentation, which used a series of morphology filtering operations to improve the segmentation, and then analyzed the connected components to obtain the desired region of interests (ROIs) [5]. Tooth isolation in upper jaw and lower jaw and the visibility of the dental plaque is improved by K-Means clustering based pseudo coloring. The tooth isolation is done by using histogram aided spectral spatial classification [6]. To identify Periodontal Diseases digital subtraction radiography (DSR) is used. It requires a pair of images with identical gray-level distribution and Projection geometry is needed for identification of periodontal disease. The drawback of this technique is if there is any mechanical alignment in the camera view then mismatching will occur in the result [7].

The classification of types of dental cyst using texture parameters estimation based on Gray Level Cooccurrence Matrix (GLCM ) approach and the K- mean classifier to classify the dental cyst based on the features. the following process can be applied to classify the dental disease. Dental cyst images are given as input then preprocessing such as image negative and special stretching is applied on image, then GLCM calulations are done and the process is followed by texture parameter estimation and lastly classification is done by using K-mean classifier [8]. Classification of dental diseases is decided on the basis of certain criteria, such as based on the lesion, whether it is within enamel, dentin or whether it touches the pulp[9].

Abdolvahab Ehsani Rad, used a digital dental radiographs to perform segmentation using Level set method and Texture Feature Extraction using gray level co-occurence matrix and also developed a system to enhance the quality of input $\mathrm{X}$-Ray image for segmentation and extacted textural features for each dental X-ray image[10]. An algorithm is developed for human identification based on SIFT feature extraction from the image.
Both radiograph and photograph are used in this system. The system developed such that, it requires less computational time and an automated approach for extracting and matching teeth features[11].

This paper gives the review on the digital image processing techniques for intraoral dental diseases. In this the review section is divided in to three subsections in which first section different image enhancement methods are discussed. the second section focuses on image segmentation and feature extraction techniques used by various authors. And A cost effective caries detection technique is developed by using image processing. It is efficient and precise in determining the tooth caries. The input image is in format of rgb. To find the caries present in the teeth the red plane is used [13].

For segmentation of dental radiographs, this method proposes the automatic selection of region of interest. The system uses binary edge intensity integral curves. to implement the automatic system it used region growing approach followed by canny edge detector. It automatically finds the ROI both for gap valley and tooth isolation [14]. A semiautomatic algorithm is proposed for measuring the root canal length based on morphological features. The basic prepocessing steps are done such as histogram equalization, denoising , andenhancement. Then morphological operations are used to extract pulp of teeth in a radiography image.Based on that root canal length is measured by giving some threshold value [15].

This paper presents an edge flow model for detection of various types of image boundaries within single framework and demonstrated the use of this model in segmenting a large variety of natural images [16].

This paper presents a very effective and fully automatic tooth isolation method which is an important pre-processing step of both computer aided dental diagnosis system and automatic dental identification system. This method contains four major steps: upper-lower jaw separation, single tooth isolation, isolation-curve verification for over segmentation, And missing-teeth detection for under segmentation. Vertical and horizontal integral projection method is used for isolation of teeth. Furthermore this can be used to indicate the locations of missing teeth [17]. A method for dental classification based on multiple fuzzy attributes for periapical radiographs is proposed. Each tooth is analyzed based on various criteria such as are/perimeter ratio, and width/height ratio. For tooth isolation integral projection is used and then features are extracted from isolated tooth such as area, perimeter, height, width etc. These features are used to classify the teeth using multiple fuzzy attributes [18]. 


\section{DISCUSSION}

The major researchers make use of thresholding and morphological operation for feature extraction and segmentation. Much of the work have been done for teeth segmentation, but very few researchers have applied and realized the methods for diagnosis purpose. Interactive portions of X-ray selected for further processing specifically for the purpose of diagnosis is the need of the hour as it would help both doctor and patient to understand the problem and depth of disease. Researchers up till now have been found concentrating on image enhancement or segmentation for extracting features for forensic sciences. No much research has effectively contributed for the diagnostic methods. Automated or semi-automated diagnosis of aforesaid objectives would be quiet useful for doctor as well as patient.

\section{REFERENCES}

[1]. Stefan Oprea, Costin Marinescu, Ioan Lita, Daniel Alexandru Visan, Ion Bogdan Cioc, "Image Processing Techniques used for Dental X-Ray Image Analysis".

[2]. Jincy Raju, Dr. Chintan K. Modi," A Proposed Feature Extraction Technique for Dental X-Ray Images Based on Multiple Features", Conference Paper July 2011 DOI: 10.1109/CSNT.2011.116 · Source:IEEE Xplore

[3]. Vijayakumari Pushparaj, Ulaganathan Gurunathan, Banumathi Arumugam, " An Effective Dental Shape Extraction Algorithm Using Contour Information and Matching by Mahalanobis Distance",Journal of Digit Imaging. 2013 Apr; 26(2):259-268.

[4]. Eyad Haj Said, Diaa Eldin M. Nassar,Gamal Fahmy, Hany H. Ammar," Teeth Segmentation in Digitized Dental X-ray Films using Mathematical Morphology", IEEE, ISSN: 1556-6013 Transactions on Information Forensics and security

[5]. Sharmila. M, Dr. R. Ganesan, R. Kartika Devi, "Detection of Dental Plaque using Image Processing", IJAIST, Vol.18, No. 18,

[6]. October 2013

[7]. M.Thamarai, M.Kalpa, "Automated Diagnosis of Periodontal Diseases Using Image Processing Techniques", IJIRSET,Vol. 3 Issue 1, January 2014
[8]. A.Farzana Shahar Banu, M. Kayalvizhi, Dr. Banumathi Arumugam, "Texture Based Classification of Dental Cysts", ICCICCT,2014

[9]. M.V. Bramhananda Reddy, VaradalaSridhar, M. Negendra, "Dental X-RayImage Analysis by using Image Processing Techniques", IJARCSSE, Vol.2,Issue 6,June 2012

[10]. Abdolvahab Ehsani Rad, Mohd Shafry Mohd Rahim, AlirezaNorouzi, "Digital Dental XRay Image Segmentation and feature Extraction", TELKOMNIKA,Vol.11, No.6, June 2013

[11]. Dipali Rindhe, Ganesh Sable, "Teeth Feature Extraction and Matching for Human Identification using SIFT Algorithm", EJAET, Vol.2 No.1, 2015

[12]. Kavindra R. Jain, Narendra C. Chauhan,"Efficacy of Digital Image Processing Techniques in intra Oral Dentistry", TROI,

[13]. Vol. 2, Issue 2, 2015

[14]. Adhar Vashishth, Bipan Kaushal, Abhishek Srivastava, "Caries Detection Techniquefor Radiographic and Intra Oral Camera",IJSCE Vol-4, Issue-2, May 2014

[15]. Chintan K. Modi and Nirav P. Desai, "A Simple and Novel Algorithm for AutomaticSelection of ROI for Dental RadiographSegmentation", IEEE CCECE 2011

[16]. Azam Amini Harandi, HosseinPourghassem, " A Semi AutomaticAlgorithm Based on Morphology Featuresfor Measuring of Root Canal Length",IEEE 2011, 978-1-61284-486$2 / 11$.

[17]. W. Y. Ma and B. S. Manjunath, "Edge Flow: A Framework of BoundaryDetection and Image Segmentation", IEEE1997, 10636919/97.

[18]. Po-Whei Huang, Phen-Lan Lin, ChunHung Kuo, Y. S. Cho, " An Effective Tooth Isolation Method for Bitewing Dental X-Ray Images ",

[19]. Martin L. Tangel, Chastine Fatichah, and Fei Yan, " Dental Classification for Periapical Radiograph based on Multiple Fuzzy Attribute", IEEE 2013, 978-1-47990348$1 / 13$. 